\title{
CHEMICALLY MODIFIED CHITOSAN COATINGS: WETTING AND ELECTROCHEMICAL STUDIES
}

\author{
PÉTER MÁRTON ${ }^{a}$, EMŐKE ALBERT'a, NORBERT NAGY ${ }^{b}$, \\ BORBÁLA TEGZEa ${ }^{a}$ GABRIELLA STEFÁNIA SZABÓ ${ }^{*}$, \\ ZOLTÁN HÓRVÖLGY|a ${ }^{\star}$
}

\begin{abstract}
Native chitosan coatings were prepared on glass and zinc substrates by dip-coating method. The native coatings were chemically modified: crosslinked with glutaraldehyde and sodium-tripolyphosphate and then silylated with dichlorodimethylsilane. The native layers prepared on zinc substrate were acylated with acetic anhydride and impregnated with indigo carmine (IC). Native coatings on different substrates showed different morphology and physical structure, which were explored by AFM studies. Examining the wetting properties of the coatings, it was found that the chemical modification can form a stable, hydrophobic (advancing contact angle of ca. $\left.97^{\circ}\right)$ and water repellent $\left(\mathrm{H}_{\odot}=2^{\circ}\right)$ layer on the glass substrate, while the same modifications reduce the hydrophobic nature and stability of the coating on zinc (contact angle decreases from ca. $100^{\circ}$ to ca. $60^{\circ}$ ). Electrochemical studies of the coatings have shown that IC increases the corrosion protection (>90\% inhibition efficiency) and reduces the permeability of the coating through ionic crosslinking, while acylation has the opposite effect and the acylated coating protection is worse than the native chitosan. The results can be used in the development of hydrophobic, water-repellent and temporary anti-corrosion coatings.
\end{abstract}

Keywords: chitosan coating, contact angles, electrochemical measurements

a Budapest University of Technology and Economics, Faculty of Chemical Technology and Biotechnology, Department of Physical Chemistry and Materials Science, Centre for Colloid Chemistry, H-1521 Budapest, Hungary

b Institute for Technical Physics and Materials Science, Centre for Energy Research, $\mathrm{H}-1525$ Budapest, Hungary

' Babeş-Bolyai University, Faculty of Chemistry and Chemical Engineering, 11 Arany Janos str., RO-400028, Cluj-Napoca, Romania

*Corresponding authors: gszabo@chem.ubbcluj.ro, zhorvolgyi@mail.bme.hu 
PÉTER MÁRTON, EMŐKE ALBERT, NORBERT NAGY, BORBÁLA TEGZE, GABRIELLA STEFÁNIA SZABÓ, ZOLTÁN HÓRVÖLGYI

\section{INTRODUCTION}

Glucosamine based biomaterials which can be frequently found in the nature are important synthesis materials for numerous products [1] [2]. Chitosan is a glucosamine based biopolymer produced from chitin [3], which is used in many industries due to its biocompatibility, biodegradability and antibacterial properties, such as food industry [4], medicine [5] and agriculture [6].

Chitosan can also be used in composite materials [7] and coatings to develop drug release systems that are very important in pharmacology. The permeability of chitosan coatings and its dependence on the layer thickness can be studied in bilayer systems [8]. The incubated molecule release kinetics is investigated from the chitosan-covered porous layer that is impregnated with the model molecule (usually an ionic dye). In this case, it is important to characterize the permeability of the chitosan coating, and one of the possible ways to do this is by determining its pseudo-porosity value from electrochemical studies, including polarization curves [9]. Due to the structure of chitosan, it can be regarded as a pseudo-porous layer through which the charged species of the electrolyte solution can reach the metal surface. Using the suitable equations, we can deduce the permeability from the rate of ion transport and the resistance of the coating.

Related to this, another area of application for chitosan is in corrosion protection [10]. Although unmodified native chitosan does not have a remarkable protective effect, it can provide effective protection when chemically modified or impregnated with various organic inhibitors. An example of such an inhibitor is indigo carmine (IC) [11], which is a natural dye and thus offers an eco-friendly alternative to more conventional corrosion inhibitors. The protective effect of these chitosan-based coatings were studied on various metallic surfaces, such as aluminum alloys [12], steel [13], zinc [14] and titanium [15]. Due to their low barrier properties, these types of chitosanbased films are often developed for less demanding applications, such as temporary protective layers. These types of coatings are capable of reducing the quantity of corrosion damage during shipment [16].

As was mentioned before, the chemical modification (e.g. impregnation with inhibitors) is a way to improve the protective effect of the coatings. If the hydrophobicity of the surface can be increased by some methods, the corrosion process can also be slowed down, as one of its primary causes is moisture. The production of hydrophobized chitosan has been reported in several articles, mainly silyl- [17] [18] or long-chain carboxylic acid derivatives [19] were used as reagents. Acylation of chitosan, which is basically a process in which chitin is produced, is also common [20] [21]. 
The aim of this paper is to investigate the effect of the mentioned chemical modifications on the wettability and pseudo-porosity (and related permeability) of chitosan coatings on various substrates. The purpose of the chemical modification with dichlorodimethylsilane (DCDMS) was to form a chitosan layer with hydrophobic and water-repellent properties. The change in the pseudo-porosity and corrosion protection of the coatings by reacting with acetic anhydride and impregnation with IC was also studied.

\section{RESULTS AND DISCUSSION}

\section{Optical spectroscopy analysis of coatings deposited on glass substrates}

The deposited layers on both substrates were transparent and completely covered the surface without any cracks or inhomogeneity. The analysis of the transmittance curves (see Fig. 1) for coatings on glass (Gls) substrates revealed layer thickness of $360 \pm 10 \mathrm{~nm}$ and a refractive index of $1.54 \pm 0.01$.

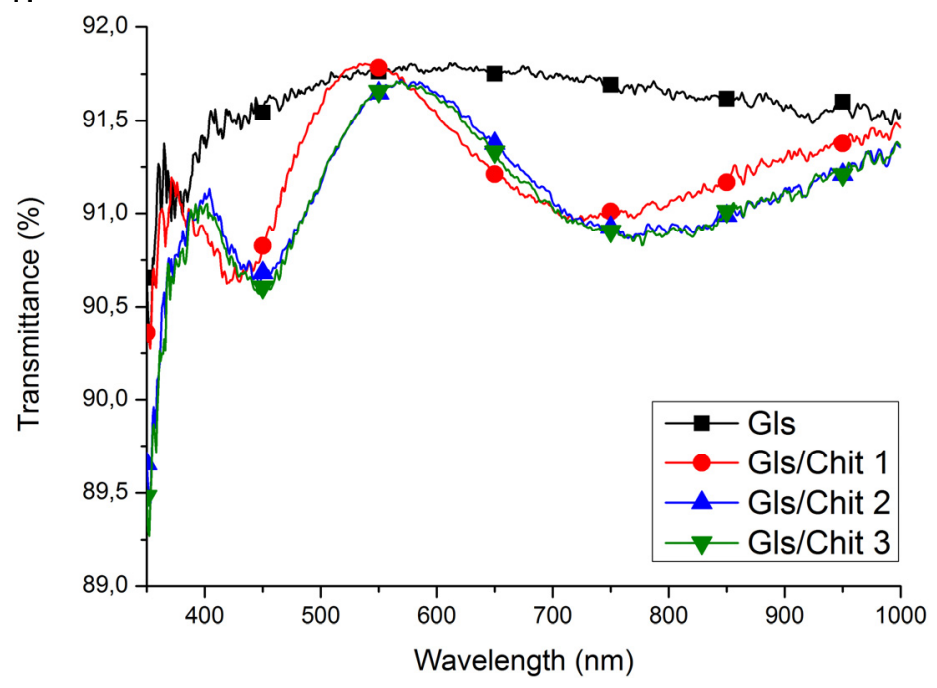

Figure 1. Transmittance spectra of the bare glass substrate and native coatings

\section{AFM measurements}

Fig. 2 shows the results of AFM measurements of the samples. The left image quartet shows the height (a) and phase image (b) of the bare glass substrate, and the height (c) and phase image (d) of the coated glass. The image quartet on the right is the same for the bare and coated zinc $(\mathrm{Zn})$. 
In the case of the bare glass substrate the surface irregularities are in the magnitude of $10 \mathrm{~nm}$, while in the case of the much rougher $\mathrm{Zn}$ substrate there are height differences of $100 \mathrm{~nm}$. The latter observation can be explained by the fact that the last step in the preparation of the metal substrates was polishing with $0.3 \mu \mathrm{m} \mathrm{Al}_{2} \mathrm{O}_{3}$ powder. It can be seen, that the surface properties of the substrates also influenced the morphology of the prepared coatings: the roughness of the chitosan layer formed on the $\mathrm{Zn}$ surface is an order of magnitude larger. In addition, a finer structure also appears in the coatings on $\mathrm{Zn}$, which is presumably not due to the roughness of the substrate but a result of the chitosan's own structure.

Studying the phase images of the samples, it can be observed that in the case of the film prepared on glass substrate, the surface is smooth, but structurally inhomogeneous: the phase image shows brighter areas (islands with stronger physicochemical interactions, marked with black arrow) with a diameter of 1-2 $\mu \mathrm{m}$. In contrast, the rougher surface on the $\mathrm{Zn}$ substrate has structural homogeneity. Presumably, the chitosan on the glass substrate separated into two domains with different surface properties before the gelation. A similar phenomenon was observed by $\mathrm{Ye}$ and Zao, who also reported in their paper the phase separation of a polymer layer [22]. It seems that the interactions between the molecules of the layer and the substrate greatly influence the structure and morphology of the final coatings.
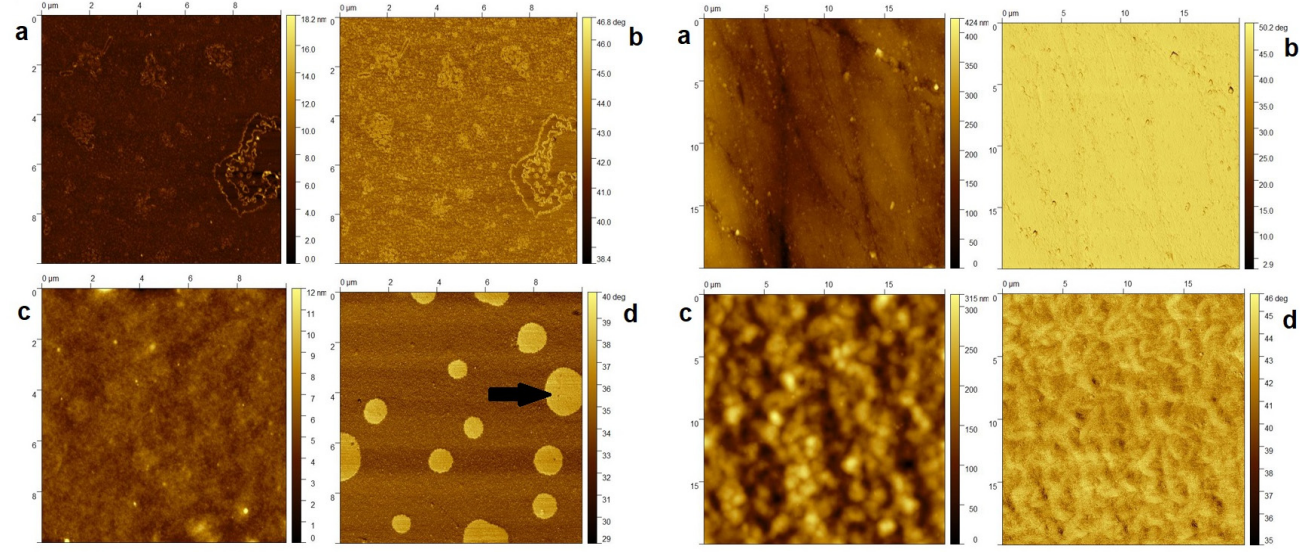

Figure 2. Results of AFM measurements: Height (a, c) and phase (b, d) images of bare $(a, b)$ and coated (c, d) glass substrates (left) and the same for $\mathrm{Zn}$ substrates (right). 


\section{Wettability of the coatings}

\section{Native coatings}

The values of the advancing and receding contact angles and the contact angle hysteresis measured on the different substrates for each measuring liquid are given in Table 1.

Table 1. Results of the wettability measurements of native coatings on both substrates $\left(\Theta_{A}\right.$ : advancing contact angle, $\Theta_{R}$ : receding contact angle, $\mathrm{H}_{\ominus}$ : contact angle hysteresis)

\begin{tabular}{|c|c|c|c|c|}
\hline \multirow{2}{*}{ Sample } & $\begin{array}{c}\text { Measuring } \\
\text { liquid }\end{array}$ & $\begin{array}{c}\boldsymbol{\Theta}_{\mathrm{A}} \\
{\left[{ }^{\circ}\right]}\end{array}$ & $\begin{array}{c}\boldsymbol{\Theta}_{\mathrm{R}} \\
{\left[{ }^{\circ}\right]}\end{array}$ & $\begin{array}{c}\mathrm{H}_{\odot} \\
{\left[{ }^{\circ}\right]}\end{array}$ \\
\hline \multirow{2}{*}{ Gls/Chit } & Distilled water & $71 \pm 9$ & $68 \pm 9$ & $4 \pm 1$ \\
\cline { 2 - 5 } & $\begin{array}{c}0.2 \mathrm{~g} / \mathrm{L} \\
\mathrm{Na}_{2} \mathrm{SO}_{4} \\
\text { solution }\end{array}$ & $79 \pm 1$ & $57 \pm 6$ & $21 \pm 5$ \\
\cline { 1 - 5 } Zn/Chit & $101 \pm 8$ & $74 \pm 10$ & $27 \pm 8$ \\
\hline
\end{tabular}

For both cases significant contact angle hysteresis was found due to the chemical heterogeneity $\left(21^{\circ}, \mathrm{Gls} / \mathrm{Chit}\right)$ or the surface roughness $\left(27^{\circ}\right.$, $\mathrm{Zn} / \mathrm{Chit}$ ). Water contact angles, however, do not show significant hysteresis for samples Gls/Chit. Water is a better wetting agent than the aqueous salt solutions [23] and the quick penetration of water molecules into the layer can result in decreased surface microheterogeneity. However, it seems from the data, that this process manifests itself in a significant macroscopic heterogeneity demonstrated by a relatively high standard deviation of contact angle data (Table 1) and a significant deterioration of water contact angles on Gls/Chit during the investigated time period of 30 minutes (Fig. 3). Macroscopic heterogeneity was also observed with aqueous salt solutions for samples $\mathrm{Zn} / \mathrm{Chit}$ but in that case the wetting stability of the chitosan layer was found to be much better (Fig. 3), which outlines the complexity of these processes.

In the case of Gls/Chit there is not much difference in the advancing contact angle values of the two measuring liquids, however, the value is below $90^{\circ}$ in both cases, which indicates the hydrophilic nature of the surface. The contact angle hysteresis is low in the case of water, but much higher in the case of the $\mathrm{Na}_{2} \mathrm{SO}_{4}$ solution, so the surface has no waterrepellent property. In the case of $\mathrm{Zn} / \mathrm{Chit}$, the advancing contact angle is well above $90^{\circ}$, but the hysteresis is also a high value, so the surface is hydrophobic, but does not show water-repellent effect. 
The change in advancing contact angles over time is shown on Fig. 3. The results show that the contact angle on the coated $\mathrm{Zn}$ surface decreases very slowly below $90^{\circ}$, so it can be considered as a hydrophobic layer that is stable in time. However, in the case of the coated glass substrate the stability of the coating is poor, the contact angle decreases significantly over time.

The contact angle data help to interpret information obtained from the analysis of the AFM images (see Fig. 2). Knowing the measured contact angles on each surface, we can assume, that that the phase which produced a larger phase shift on the AFM images belongs to a more nonpolar structure, and on the $\mathrm{Zn}$ surface only that structure was formed. However, on the glass substrate a heterogeneous surface was formed (Fig. 2c) with smaller nonpolar domains (producing the same phase shift) surrounded by an area of presumably more polar character (producing a different, lower phase shift). These two phases together determine the wetting properties of the coating formed on glass. Knowing the value of the advancing contact angle of the $\mathrm{Na}_{2} \mathrm{SO}_{4}$ solution measured on the nonpolar surface ( $\mathrm{Zn} / \mathrm{Chit})$, we can apply the Cassie-Baxter wetting model to determine the value of the advancing contact angle of the $\mathrm{Na}_{2} \mathrm{SO}_{4}$ solution on the polar phase formed on the glass substrate. The essence of the model is that on a heterogeneous surface consisting of areas with different wettability, the measurable contact angle can be calculated by a linear combination of the contact angles of each type of area [24].

$$
\cos \Theta_{o b s}=f \cdot \cos \Theta_{1}+(1-f) \cdot \cos \Theta_{2}
$$

where $\Theta_{o b s}$ is the value of the observed contact angle $\left(79^{\circ}\right), \Theta_{1}\left(101^{\circ}\right)$ and $\Theta_{2}$ are the contact angles characterizing the nonpolar and polar domains of the surface, and $f$ is the percentile area ratio of the nonpolar domains. This $f$ parameter can be determined from the AFM phase images: $12 \%$. The resulting contact angle value $\left(\Theta_{2}\right)$ for the polar part of the surface is $76^{\circ}$. This is not a big difference from the measurable value but the presence of the nonpolar domains can cause significant contact angle hysteresis as was mentioned above.

Thus, it can be seen that coatings of the same material prepared with the same technique on the various surfaces show greatly different wettability behavior. This may be explained by the different chemical and physical nature of the $\mathrm{Zn}$ and glass substrates which can influence the structure (surface polarity) and morphology of the deposited layers. 


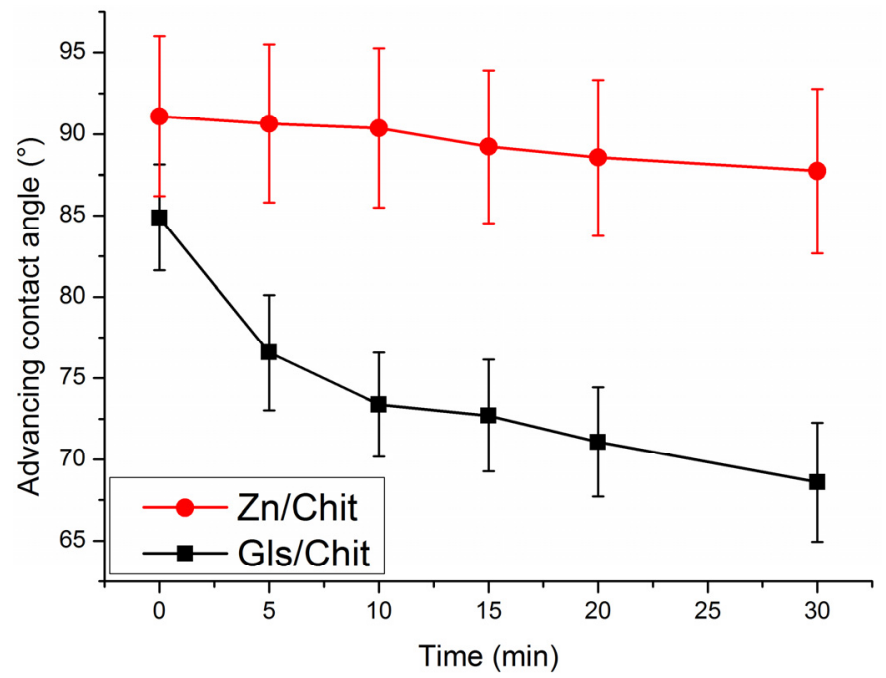

Figure 3. Change in advancing contact angle values over time for coatings on both substrates (In the case of glass substrates, the measurement was performed with distilled water, in the case of $\mathrm{Zn}$ substrates with $0.2 \mathrm{~g} / \mathrm{L} \mathrm{Na}{ }_{2} \mathrm{SO}_{4}$ solution).

\section{Silylated coatings}

\section{Preliminary investigations for the modified chitosan layers deposited on glass substrates}

Fig. 4 shows the water droplet shapes and relating advancing contact angles on silylated chitosan coatings on glass substrates, measured $3,5,10$, 20 and 40 seconds after the drop formation. For the native coating (Fig. 4a), the contact angles reach a relatively constant value (ca. $75^{\circ}$ ) after $10 \mathrm{~s}$ (also see Table 1). For the sample silylated without crosslinking (Fig. 4b), the contact angle initially exceeds $90^{\circ}$, but rapidly decreases below the value measured on the native coating. The same is observed for the coating crosslinked with pentasodium-tripolyphosphate (TPP), then silylated (Fig. 4c), but the decrease is smaller. However, the sample crosslinked with glutaraldehyde (GDA), then silylated (Fig. 4d) keeps a contact angle above $90^{\circ}$ in the investigated 5minute time range.

For interpreting the above observations, it should be taken into consideration that the silylation reaction with DCDMS produces $\mathrm{HCl}$, which diffuses into the layer. When the droplet is placed onto the surface, in the aqueous medium the acid can protonate the free amino groups of chitosan forming a much more polar (i.e. hydrophilic) coating. In addition, the diffusion of the hydrophilic parts of the polymer chains to the surface become faster in 
the aqueous medium (Fig. 4b). The crosslinkers, however, react with the amino groups of chitosan: TPP forms ionic bonds between the protonated amino groups in aqueous solution, and GDA is covalently bound to the nitrogen atoms. In both cases, the basicity of the amino groups decreases significantly, and the $\mathrm{HCl}$ is no longer able to protonate them, so that the contact angle does not decrease to the previously seen extent (Fig. 4c and d). In addition, crosslinking reduces the mobility of the polymer chains upon contact with the aqueous phase, therefore the hydrophobic silyl groups can stay on the surface while maintaining their effect. The results show that the covalently crosslinking agent (GDA) is better than the other one: temporal stability was only experienced for the case of GDA crosslinking (Fig. 4d).

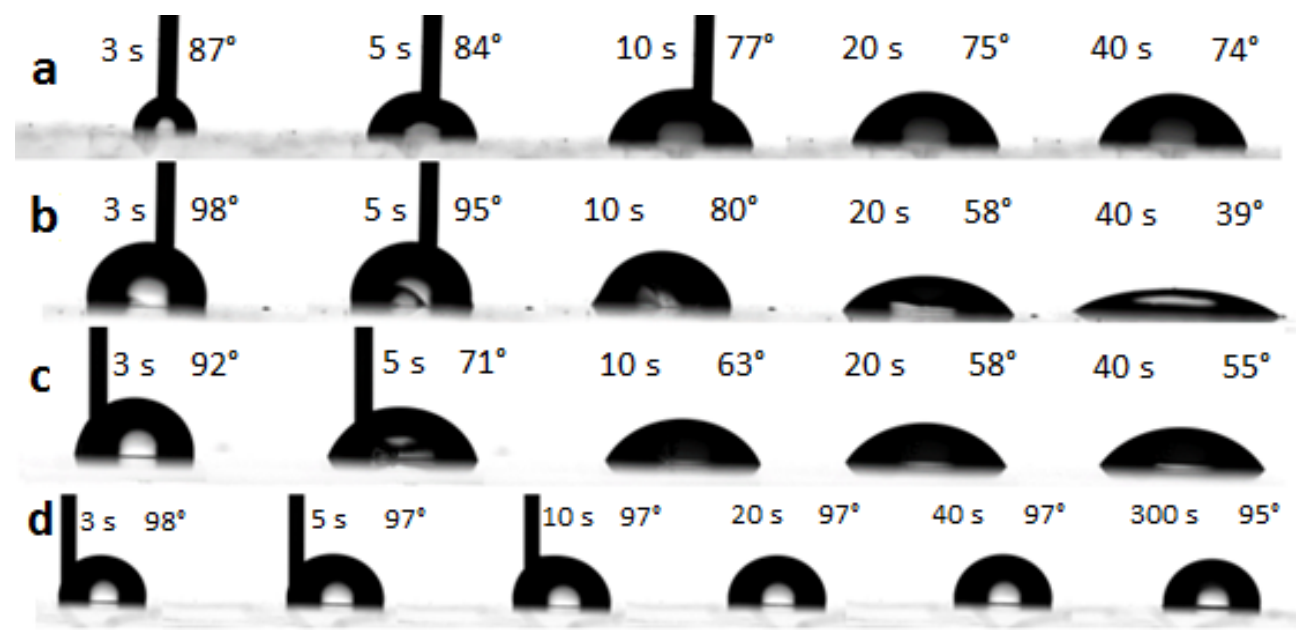

Figure 4. Water droplets on different surfaces: advancing contact angles at the indicated time after droplet formation (a: native chitosan, b: chitosan, silylated with

DCDMS, c: chitosan, cross-linked with TPP, silylated with DCDMS d: chitosan, cross-linked with GDA, silylated with DCDMS)

\section{Comparative investigation of the wetting properties of modified chitosan layers deposited on glass and $\mathrm{Zn}$ substrates}

The advancing and receding water and $\mathrm{Na}_{2} \mathrm{SO}_{4}$ solution contact angles measured for the GDA-crosslinked and DCDMS-modified samples on glass (Gls/Chit-GDA-Silyl) and Zn substrates (Zn/Chit-GDA-Silyl) are shown in Table 2. All samples were silylated in the vapour phase of the $n$-hexane solution of DCDMS. Comparing the data in Tables 1 and 2, it can be observed that in the case of Gls/Chit-GDA-Silyl, the silylation improved the water-repellent 
properties of the coating: the water contact angle is above $90^{\circ}$ and the value of the hysteresis is very small, which means that the surface is hydrophobic and water-repellent. The water contact angles show a relatively good stability in the investigated time range (Fig. 5a). It is important to note, however, that the samples lose their advantageous properties by soaking them for 10 minutes in water: the value of the water contact angles decrease below the values measured on the native chitosan. However, the silylation improved the wettability of the chitosan layer on the $\mathrm{Zn}$ substrate compared to the wettability of the native chitosan layer, and the wetting stability also deteriorated (Fig. 5b). Interestingly, by increasing the time of silylation the resulting water contact angles will be even smaller (Fig. $5 b$ ).

The different behaviour of chitosan coatings on the glass and $\mathrm{Zn}$ substrates during the cross-linking and silylation processes can be attributed to the different polarity of the pertinent native coatings. During the crosslinking process the hydrophilicity of the native coatings on glass can further increase due to the chemisorption of GDA [8]. Furthermore, as this process is carried out in aqueous phase, the reorientation of the polymer segments is facilitated and this results in the increase of the surface concentration of hydroxyl groups. The efficiency of the silylation can be attributed to the latter process. The reorientation process is presumably hindered for coatings on $\mathrm{Zn}$ substrates due to the hydrophobic character of the surface. Water molecules cannot penetrate the outside of the layer fast enough and so the mobility of the polymer segments is lower. Hence, in this case the surface polarity increase can only happen due to chemisorption of GDA.

Table 2. Wettability results of coatings cross-linked with GDA and silylated with DCDMS under various conditions ( ${ }^{*}$ after 10 min swelling in distilled water and drying) $\left(\Theta_{A}\right.$ : advancing contact angle, $\Theta_{R}$ : receding contact angle, $\mathrm{H}_{\odot}$ : contact angle hysteresis)

\begin{tabular}{|c|c|c|c|c|}
\hline Sample & $\begin{array}{c}\text { Silylation } \\
\text { time }\end{array}$ & $\begin{array}{c}\boldsymbol{\Theta}_{\mathbf{a}} \\
{\left[{ }^{\circ}\right]}\end{array}$ & $\begin{array}{c}\Theta_{\mathrm{r}} \\
{\left[{ }^{\circ}\right]}\end{array}$ & $\begin{array}{c}\mathrm{H}_{\odot} \\
{\left[{ }^{\circ}\right]}\end{array}$ \\
\hline \multirow{2}{*}{ Gls/Chit-GDA-Silyl } & \multirow{2}{*}{$30 \mathrm{~min}$} & $97 \pm 1$ & $95 \pm 3$ & $2 \pm 2$ \\
\cline { 2 - 5 } & & $48 \pm 2^{*}$ & $31 \pm 8^{*}$ & $16 \pm 6^{*}$ \\
\hline \multirow{2}{*}{ Zn/Chit-GDA-Silyl } & $5 \mathrm{~min}$ & $77 \pm 15$ & $57 \pm 24$ & $20 \pm 13$ \\
\cline { 2 - 5 } & $10 \mathrm{~min}$ & $64 \pm 10$ & $47 \pm 13$ & $23 \pm 5$ \\
\hline
\end{tabular}



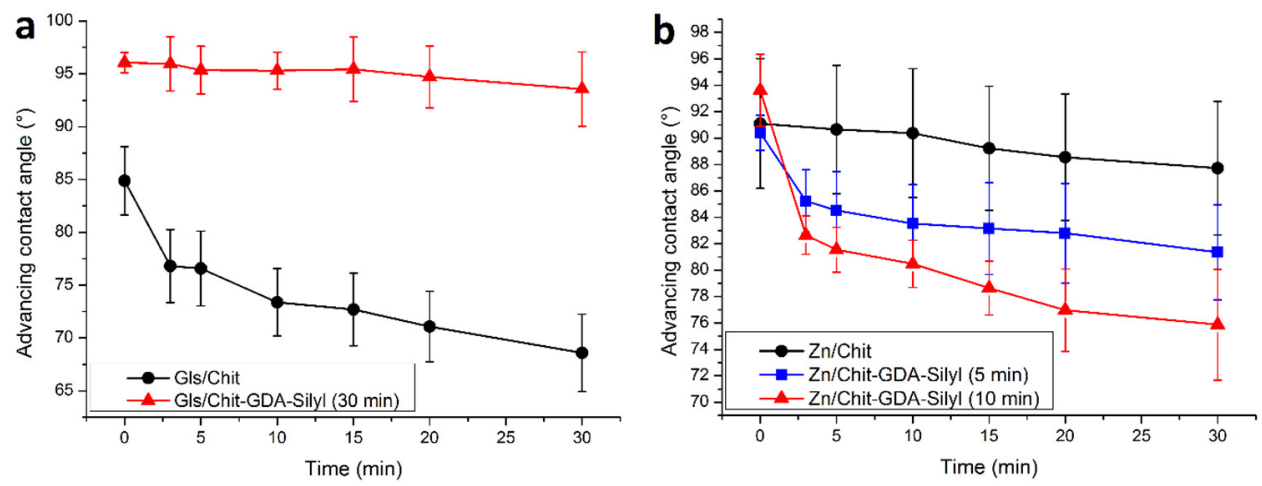

Figure 5. Advancing contact angle values measured in time for chemically modified (cross-linked with GDA and silylated with DCDMS) coatings on glass (a) and $\mathrm{Zn}$ (b) substrates. (In the case of glass substrates, the measurement was performed with distilled water, in the case of $\mathrm{Zn}$ substrates with $0.2 \mathrm{~g} / \mathrm{L} \mathrm{Na} 2 \mathrm{SO}_{4}$ solution).

\section{Electrochemical measurements of the coatings}

The linear and semi-logarithmic polarization curves for the investigated samples are represented in Fig. 6.
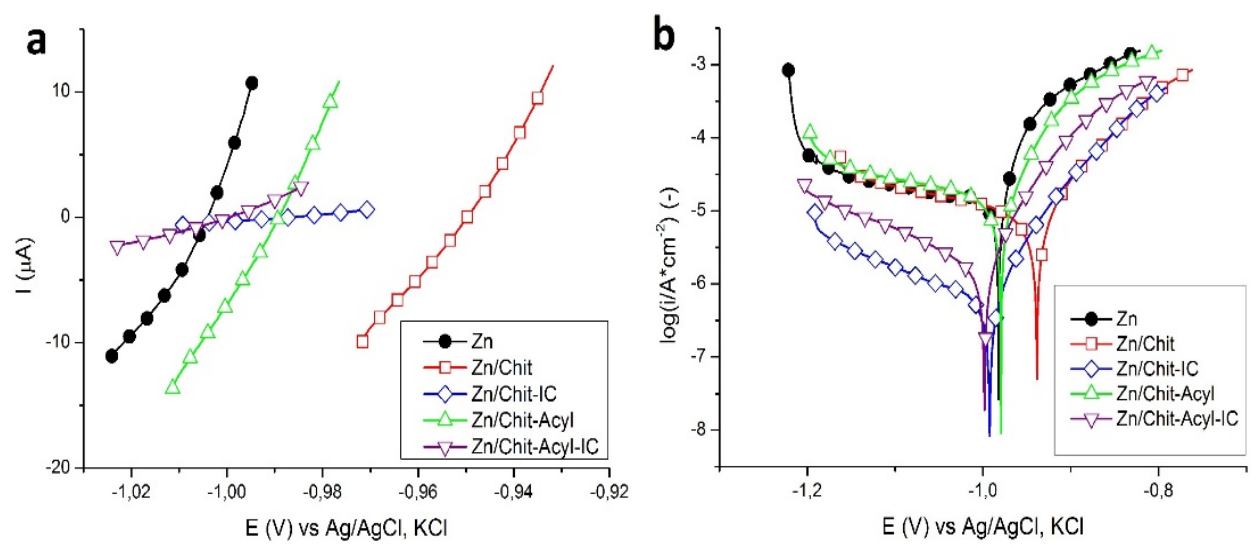

Figure 6. Linear (a) and semi-logarithmic (b) polarization curves for the bare $\mathrm{Zn}$ and the coated samples

In the Table 3. are introduced the OCP, the slopes of the fitted straight lines to the linear polarization curves and the calculated polarization resistance $R_{p}$. This is the inverse value of the straight line's slope, that was fitted to the linear portion of the curves. Further characteristics of the layers 
like pseudo-porosity $(\mathrm{P})$ can be calculated from the obtained data, using the following formula, assuming that the layer is electrochemically inert at low overpotentials [25]:

$$
P=\frac{R_{p_{s}}}{R_{p}} \cdot 10^{-\left(\frac{\left|\Delta E_{c o r r}\right|}{b_{a}}\right)} \cdot 100(\%)
$$

The $R_{p s}$ and $R_{p}$ represent the polarization resistance of the bare $\mathrm{Zn}$ substrate and the coated $\mathrm{Zn}$, respectively, while $\Delta \mathrm{E}_{\text {corr }}$ symbolizes the difference between the potential of the coated and uncoated samples, and $b_{a}$ is the anodic Tafel coefficient of the bare substrate.

Table 3. The polarization resistance $R_{p}$ : determined from the linear polarization curves recorded for the bare $\mathrm{Zn}$ and the coated samples. B represents the slope of the fitted line, $R^{2}$ the coefficient of determination, $N$ the number of the points, OCP the open circuit potential and P the pseudo-porosity

\begin{tabular}{|c|c|c|c|c|c|c|}
\hline Sample & $\begin{array}{c}\mathbf{R}^{2} \\
{[-]}\end{array}$ & $\begin{array}{c}\mathbf{N} \\
{[-]}\end{array}$ & $\begin{array}{c}\mathbf{1 0}^{4} \cdot \mathbf{B} \\
{[\mathbf{1 / \Omega}]}\end{array}$ & $\begin{array}{c}\mathbf{R}_{\mathbf{p}} \\
{[\mathbf{\Omega}]}\end{array}$ & $\begin{array}{c}\mathbf{P} \\
{[\%]}\end{array}$ & $\begin{array}{c}\text { OCP } \\
{[\mathbf{V}]}\end{array}$ \\
\hline Zn & 0.999 & 25 & 7.31 & 1368 & - & -0.987 \\
\hline Zn/Chit & 0.999 & 27 & 4.67 & 2141 & 44 & -0.944 \\
\hline Zn/Chit-IC & 0.999 & 33 & 0.28 & 35714 & 4 & -0.977 \\
\hline Zn/Chit-Acyl & 0.999 & 27 & 6.56 & 1524 & 88 & -0.973 \\
\hline Zn/Chit-Acyl-IC & 0.998 & 23 & 1.63 & 6135 & 19 & -0.999 \\
\hline
\end{tabular}

If the value of the polarization resistance is high, the coating has a good anti-corrosion property. Vice versa if it is low, protective property of the coating is poor. As one can see from Table 3, the highest corrosion resistance $R_{p}$ is attributed to the Chit-IC layer, indicating the best anticorrosion effect. The corrosion current densities are proportional to the corrosion rate, which means that this value is low in the case of a good protective coating. In Table 4 the lowest value is attributed to the Chit-IC coating, this has the best anticorrosion property, which is in a very good agreement with the $R_{p}$ obtained for this layer from the linear polarization.

The low porosity of the Chit-IC coating is correlated with a relatively denser texture. 
PÉTER MÁRTON, EMŐKE ALBERT, NORBERT NAGY, BORBÁLA TEGZE, GABRIELLA STEFÁNIA SZABÓ, ZOLTÁN HÓRVÖLGYI

Table 4. Polarization parameters obtained by Tafel interpretation of the semi-logarithmic polarization curves: icorr: corrosion current density,

$E_{\text {corr }}$ corrosion potential, $b_{a}$ and $b_{c}$ anodic and cathodic

Tafel coefficients. IE represent the inhibition efficiency

\begin{tabular}{|c|c|c|c|c|c|}
\hline Sample & $\begin{array}{c}\mathbf{i}_{\text {corr }} \\
{\left[\mu \mathbf{A} / \mathbf{c m}^{2}\right]}\end{array}$ & $\begin{array}{c}\mathbf{E}_{\text {corr }} \\
{[\mathbf{V}]}\end{array}$ & $\begin{array}{c}\mathbf{b}_{\mathbf{a}} \\
{[\mathbf{V} / \mathbf{d e c}]}\end{array}$ & $\begin{array}{c}\mathbf{b}_{\mathbf{c}} \\
{[\mathbf{V} / \mathbf{d e c}]}\end{array}$ & $\begin{array}{c}\mathbf{I E} \\
{[\%]}\end{array}$ \\
\hline Zn & 8.945 & -0.982 & 0.272 & 0.029 & - \\
\hline Zn/Chit & 5.667 & -0.939 & 0.144 & 0.057 & 36.6 \\
\hline Zn/Chit-IC & 0.388 & -0.992 & 0.040 & 0.105 & 95.7 \\
\hline Zn/Chit-Acyl & 7.064 & -0.979 & 0.036 & 0.097 & 21.0 \\
\hline Zn/Chit-Acyl-IC & 0.688 & -0.999 & 0.067 & 0.209 & 92.3 \\
\hline
\end{tabular}

For the calculation of the inhibition efficiency (IE) formula (3) is used:

$$
I E=\frac{i_{\text {corr }}^{s}-i_{\text {corr }}}{i_{\text {corr }}^{s}} \cdot 100
$$

where $\mathrm{i}_{\text {corr }}{ }^{\mathrm{s}}$ and $\mathrm{i}_{\text {corr }}$ are the corrosion current densities of the $\mathrm{Zn}$ substrate and the coated sample, respectively. From the IE value calculated for the native chitosan (Chit) coating, 36,6\%, it can be deduced that the native chitosan layer has a very poor anti-corrosion effect. In the case of IC impregnated chitosan coating (Chit-IC) the corrosion current density decreased with one order of magnitude while the IE increase significantly $(95,7 \%)$. This means that this kind of coating has a good anti-corrosion property. The acylation of both the native Chit and Chit-IC causes the decay of the anti-corrosion properties, namely the increase of the corrosion current density and the decrease of the IE. Altough in the case of the acylated, impregnated chitosan coating (Chit-Acyl-IC) the $i_{\text {corr }}$ is better than in the case of native chitosan, still the Chit-IC has the best IE.

Considering also the calculated pseudo-porosity values from Table 3, one can say that this kind of difference in behavior is due to the permeability of the thin layers. It can be seen, that the Chit-IC has the smallest porosity and for this reason has the best anti-corrosion property. The increasing order of the porosity in case of the other coatings is Chit-Acyl-IC, Chit and at last the Chit-Acyl with a very high value (88\%).

The explanation of this phenomenon lays in the structure of the coatings. The IC molecules contain negatively charged groups and can crosslink the chitosan, forming ionic bonds with the protonated amino groups. The layer formed in this way has a reduced permeability. By acylation, the number 
of the amino groups that can be protonated is significantly reduced, subsequently the extent of the crosslinked portions are reduced too and the permeability against the corrosive environment is increased. In this way the anti-corrosion properties of the coating are diminished. It can be assumed, that as a consequence of the acylation $\mathrm{H}$ bonds also cannot be formed between the amino groups. This could be the reason why the acylated coating protection is worse that even that of the native chitosan. The sterical effect of the acyl-groups can also be the cause of weaker structure formation. The acylating reaction conditions can induce the damage of the materials.

\section{CONCLUSIONS}

Wetting and electrochemical properties of chemically modified chitosan coatings prepared on glass and zinc substrates were investigated. The purpose of the research was to develop a water-repellent and corrosion protective polymer layer.

Wettability studies of native coatings have shown that the chitosan layer prepared on glass substrate is hydrophilic and unstable in time, while on the zinc substrate a highly hydrophobic and stable in time but non-waterrepellent coating is formed under the same conditions. AFM analysis of the layers detected surfaces with different morphologies and signs of phase separation of chitosan on the glass substrate.

Examination of the wettability of the silylated coatings revealed that of the systems studied, only the samples crosslinked with GDA and then chemically modified with DCDMS provided a surface with satisfactory properties. In this way, a coating with hydrophobic and water-repellent properties, which is very stable in time, has been produced on a glass substrate, however, on zinc substrates the silylation greatly deteriorates the properties of the originally hydrophobic layer.

Electrochemical studies have shown that among the chemical modification methods studied, impregnation with IC improves the anticorrosion effect of chitosan coatings which is explained by the cross-linking effect of IC. The acylation, however, greatly diminished the corrosion protection by increasing the pseudo-porosity of the coatings.

\section{EXPERIMENTAL SECTION}

MilliQ water (Millipore Simplicity 185, Resistance: 18,2 M $\Omega \mathrm{cm}$ ); sulfuric acid (Carlo Erba, 96\%, f. a.); isopropanol (Reanal Labor, 99,7\%, f. a.); acetic acid (Lachner, 99,8\% f. a.); chitosan (Sigma-Aldrich, Medium molecular weight, Viscosity: $598 \mathrm{cP}$ ); glutaraldehyde (Reanal Labor, 25\%, purum); 
pentasodium-tripolyphosphate-pentahydrate (Sigma-Aldrich, $\geq 98 \%$, purum); dichlorodimethylsilane (Reanal Labor, $\geq 99 \%$, f. a.); n-hexane (Merck, 95\%, f. a.); indigo carmine (Reanal Labor, f. a.); acetic anhydride (Reanal, f. a.); methanol (Molar Chemicals Kft.).

The coatings were prepared on glass microscope slides (MenzelGläser) and zinc plates (Bronzker Bt.) with a dip coater made by Plósz Engeneering Ltd.

\section{Coatings preparation}

The glass substrates were cleaned using aqueous detergent solution, $10 \%(\mathrm{w} / \mathrm{w})$ sulfuric acid, isopropanol and MilliQ water. The $\mathrm{Zn}$ substrates were polished with emery paper (P1200, P2000, and P3000) and $\mathrm{Al}_{2} \mathrm{O}_{3}$ powder (particle size: $0,3 \mu \mathrm{m}$ ). Then they were washed with isopropanol and MilliQ water and ultrasonicated for 10 min to remove any powder residue. Before the dip-coating, $0.1 \mathrm{M} \mathrm{HCl}$ solution was used to clean the surface of any remaining zinc-oxide.

The dip-coating was performed with a withdrawal speed of $5 \mathrm{~cm} / \mathrm{min}$ from $1 \%(\mathrm{w} / \mathrm{w})$ chitosan solution. The chitosan was dissolved in $1 \%(\mathrm{v} / \mathrm{v})$ acetic acid solution, stirred for 24 hours, then centrifuged (30 min, $4000 \mathrm{rpm}$ ) to remove any insoluble chitosan residue due to imperfect deacetylation. The prepared coatings were dried overnight at room temperature.

\section{Chemical modification of the coatings}

Some samples were chemically modified by acylation and/or impregnation with IC. Other samples were modified by cross-linking agents and/or silylation.

The acylation of the coatings was carried out in $10 \%(\mathrm{v} / \mathrm{v})$ methanolbased acetic anhydride solution for $60 \mathrm{~min}$. Afterwards, the samples were washed with methanol and dried at room temperature.

The impregnation was performed using the dip-coater: the samples were immersed in $0.01 \mathrm{M} \mathrm{IC}$ solution with $10 \mathrm{~cm} / \mathrm{min}$ immersion speed, kept in the solution for $15 \mathrm{~min}$, then withdrawn with $10 \mathrm{~cm} / \mathrm{min}$.

Two different cross-linking agents (covalent, ionic) were tried. The coatings on glass substrates were covalently cross-linked in $5 \%(\mathrm{w} / \mathrm{w})$ aqueous glutaraldehyde solution for $5 \mathrm{~min}$, while the coatings on $\mathrm{Zn}$ substrates were cross-linked in 1\% (w/w) glutaraldehyde solution for $30 \mathrm{~min}$, because the more concentrated solution damaged the coating on $\mathrm{Zn}$. The ionic crosslinking by pentasodium-tripolyphosphate took place in a $3 \%(\mathrm{w} / \mathrm{w})$ aqueous solution for $10 \mathrm{~min}$. Then the samples were washed with distilled water and dried at room temperature. 
For the silylation reaction the samples were kept in a desiccator. The silylating agent was an $\mathrm{n}$-hexane solution of dichlorodimethylsilane. For the samples on glass $10 \%(\mathrm{w} / \mathrm{w})$ solution and a reaction time of $30 \mathrm{~min}$ were used; while for the samples on $\mathrm{Zn} 5 \%$ (w/w) n-hexane solution with reaction times of 5 and 10 min were used. Afterwards, the samples were kept at room temperature in air for 10 min to remove any left-over reagent.

\section{Transmittance measurements}

The layer thickness and refractive index values of the coatings on glass substrates were determined by a thin-layer optical model [26] fitted to the transmittance spectra. The transmittance was measured using an Analytik Jena Specord 200 UV-Vis spectrophotometer in the 350-900 nm wavelength range with $1 \mathrm{~nm}$ resolution and a measurement speed of $10 \mathrm{~nm} / \mathrm{s}$.

\section{AFM measurements}

To explore surface properties, the samples were measured with an AIST-NT SmartSPM 1000 AFM device in tapping mode with a PPP-NCHR20 needle made by NanoSensors (nominal radius of the needle $<20 \mathrm{~nm}$ ). In the range of $10 \times 10$ and $20 \times 20 \mu \mathrm{m}$ a height and phase image of the surfaces were recorded.

\section{Wettability measurements}

Contact angle measurements were performed on the native and on the silylated coatings deposited on glass and $\mathrm{Zn}$ substrates to get information about the effect of chemical modification on the surface polarity. The measurements were carried out with $0.2 \mathrm{~g} / \mathrm{L}$ aqueous $\mathrm{Na}_{2} \mathrm{SO}_{4}$ solution, which was also used as electrolyte during electrochemical investigations. MilliQ water was also used for the characterization of coatings deposited on glass substrates. Wettability was studied by a Drop Shape Analysis System (DSA30, Krüss GmbH). Advancing contact angles were measured by the sessile drop method by the drop-build up technique at $25^{\circ} \mathrm{C}$ and $90 \%$ humidity within the first minute after the droplet formation $(20 \mu \mathrm{l})$. Then receding contact angles were also measured after removing ca. half the volume of the droplet. Contact angle hysteresis was determined as the difference of the advancing and receding angles. In certain cases, the advancing contact angles of $20 \mu \mathrm{l}$ droplets were studied during a 30 minute time interval in order to characterize the stability of coatings. On some selected samples the contact angle values were determined after they were soaked in distilled water for $10 \mathrm{~min}$ and then dried. 
PÉTER MÁRTON, EMŐKE ALBERT, NORBERT NAGY, BORBÁLA TEGZE, GABRIELLA STEFÁNIA SZABÓ, ZOLTÁN HÓRVÖLGYI

\section{Electrochemical measurements}

The corrosion resistance of the coatings was investigated in an undivided electrochemical cell containing three electrodes, connected to the Metrohm Autolab PGSTAT 301N potentiostat. The coated Zn samples were used as working electrode, with a $2 \mathrm{~cm}^{2}$ active surface area, while the Platinum wire and the $\mathrm{Ag} / \mathrm{AgCl}, \mathrm{KCl}$ (saturated) were the counter and the reference electrode, respectively. The aggressive environment consisted of a $\mathrm{Na}_{2} \mathrm{SO}_{4}$ solution with $0.2 \mathrm{~g} / \mathrm{L}$ concentration. All measurements were carried out at room temperature.

First, for each sample the open circuit potential (OCP) was determined, and every measurement lasted for $60 \mathrm{~min}$. The potentiodynamic polarization was performed in a potential range of $\mathrm{OCP} \pm 20 \mathrm{mV}$ and $\mathrm{OCP} \pm 200 \mathrm{mV}$ vs $\mathrm{Ag} / \mathrm{AgCl}, \mathrm{KCl}$ (saturated) electrode and the obtained curves were recorded with $0.166 \mathrm{mV} / \mathrm{s}$ rate. By Tafel interpretation of the semi-logarithmic polarization curves, namely by fitting straight lines to the anodic and cathodic branches, kinetic parameters (corrosion current densities, corrosion potentials, Tafel coefficients) were determined.

\section{ACKNOWLEDGMENTS}

The research reported in this paper was supported by the BME Nanotechnology and Materials Science TKP2020 IE grant of NKFIH Hungary (BME IE-NAT TKP2020). The research work has been accomplished in the framework of the "BME R+D+I project", supported by the grant TÁMOP 4.2.1/B09/1/KMR-2010-0002. NRDI TNN-123631, NRDI K-128266, and NRDI FK128901 grants are acknowledged. Emőke Albert's research work was supported by the European Union and the State of Hungary, co-financed by the European Social Fund in the framework of TÁMOP-4.2.4.A/2-11/1-2012-0001 "National Excellence Program"

\section{REFERENCES}

1. Z. Sebestyén, E. Jakab, A. Domán, P. Bokrossy, I. Bertóti, J. Madarász, K. László, J. Therm. Anal. Calorim. 2020, 1-8.

2. B. Duan, Y. Huang, A. Lu, L. Zhang, Prog. Polym. Sci. 2018, 82, 1-33.

3. S. Kumari, S.H. Kumar Annamareddy, S. Abanti, P. Kumar Rath, Int. J. Biol. Macromol. 2017, 104 1697-1705. 
4. S. Tripathi, G.K. Mehrotra, P.K. Dutta, Int. J. Biol. Macromol. 2009, 45, 372376.

5. T. Dai, M. Tanaka, Y.Y. Huang, M.R. Hamblin, Expert Rev. Anti. Infect. Ther. 2011, 9 857-879.

6. P.L. Kashyap, X. Xiang, P. Heiden, Int. J. Biol. Macromol. 2015, 77, 36-51

7. T. V. Podust, T. V. Kulik, B.B. Palyanytsya, V.M. Gun'Ko, A. Tóth, L. Mikhalovska, A. Menyhárd, K. László, Appl. Surf. Sci. 2014, 320, 563-569.

8. M. Dabóczi, E. Albert, E. Agócs, M. Kabai-Faix, Z. Hórvölgyi, Carbohydr. Polym, 2016, 136, 137-145.

9. C. Liu, Q. Bi, A. Leyland, A. Matthews, Corros. Sci. 2003, 45, 1257-1273

10. M.L. Zheludkevich, J. Tedim, C.S.R. Freire, S.C.M. Fernandes, S. Kallip, A. Lisenkov, A. Gandini, M.G.S. Ferreira, J. Mater. Chem. 2011, 21, 4805-4812.

11. Á.F. Szőke, G.S. Szabó, Z. Hórvölgyi, E. Albert, L. Gaina, L.M. Muresan, Carbohydr. Polym. 2019, 215, 63-72.

12. O. Lundvall, M. Gulppi, M.A. Paez, E. Gonzalez, J.H. Zagal, J. Pavez, G.E. Thompson, Surf. Coatings Technol. 2007, 201 5973-5978.

13. S.A. Umoren, M.J. Banera, T. Alonso-Garcia, C.A. Gervasi, M. V. Mirífico, Cellulose, 2013, 20 2529-2545.

14. Á.F. Szőke; G. Szabó; Z. Simó; Z. Hórvölgyi; E. Albert; A.G. Végh; L. Zimányi; L.M. Muresan; Eur. Polym. J., 2019, 118, 205-212.

15. J. Redepenning; G. Venkataraman; J. Chen; N. Stafford; J. Biomed. Mater. Res. - Part A., 2003, 66, 411-416.

16. J. Carneiro; J. Tedim; S.C.M. Fernandes; C.S.R. Freire; A. Gandini; M.G.S. Ferreira; M.L. Zheludkevich; ECS Electrochem. Lett., 2013, 2, C19.

17. K. Kurita, M. Hirakawa, S. Kikuchi, H. Yamanaka, J. Yang, Carbohydr. Polym. 2004, 56, 333-337.

18. A. Tiwari, J. Inorg. Organomet. Polym. Mater. 2009, 19, 361-366.

19. S. Höhne, R. Frenzel, A. Heppe, F. Simon, Biomacromolecules. 2007, 8, 20512058.

20. J. Xu, S.P. McCarthy, R.A. Gross, D.L. Kaplan, Macromolecules. 1996, 29, 3436-3440.

21. K.Y. Lee, W.S. Ha, W.H. Park, Biomaterials. 1995, 16, 1211-1216.

22. Z. Ye, X. Zhao, J. Microsc. 2010, 238, 27-35.

23. Z. Hórvölgyi, M. Máté, M. Zrínyi, Colloids Surfaces A Physicochem. Eng. Asp. 1994, 84, 207-216.

24. E. Bormashenko, Colloids Surfaces A Physicochem. Eng. Asp. 2008, 324, 4750.

25. B. Matthes, E. Broszeit, J. Aromaa, H. Ronkainen, S.P. Hannula, A. Leyland, A. Matthews, Surf. Coatings Technol. 1991, 49, 489-495.

26. E. Hild, A. Deák, L. Naszályi, Ö. Sepsi, N. Ábrahám, Z. Hórvölgyi, J. Opt. A Pure Appl. Opt. 2007, 9, 920-930. 
